\title{
The role of myocardial perfusion imaging in the identification of the obstructive coronary artery lesions: a tertiary cardiology center experience
}

\author{
Göktuğ Savaş ${ }^{1 \oplus,}$ Melek Süzer Aslan²®, Mehmet Fatih Fırat ${ }^{3} \oplus$, Sait Terzi $^{1}{ }^{\circledR}$
}

${ }^{1}$ Department of Cardiology, Siyami Ersek Thoracic and Cardiovascular Surgery Training and Research Hospital, Istanbul, Turkey ${ }^{2}$ Department of Coronary Care Unit, Siyami Ersek Thoracic and Cardiovascular Surgery Training and Research Hospital, Istanbul, Turkey

${ }^{3}$ Department of Nuclear Medicine, Siyami Ersek Thoracic and Cardiovascular Surgery Training and Research Hospital, Istanbul, Turkey

\section{ABSTRACT}

Objectives: Because of the moderate accuracy of treadmill electrocardiogram and fear of a claim if a diagnosis is missed, cardiologists usually order Myocardial Perfusion Imaging (MPI) as a first-step test in most of patients with chest pain admitted to cardiology department. The performance of the MPI in diagnosing obstructive CAD depends on the population studied. Thus we aimed to assess the agreement between MPI and coronary angiography in the identification of the obstructive coronary lesion.

Methods: A total of 231 patients who underwent MPI due to suspicion of coronary ischemia and had a coronary angiogram within the last three months were included in this retrospective study. MPI and coronary angiography findings were analyzed to weigh the performance of MPI in determining obstructive coronary lesion.

Results: The mean age was $63.9 \pm 8.9$ years, $54.5 \%$ being males. MPI showed a sensitivity of 0.86 in determining patients who had a significant $(>70 \%)$ coronary lesion. While evaluating the ability of MPI to detect ischemia in the left ventricle region which is supplied by the lesioned vessel, the sensitivity was found to be; $60 \%$ in determining anterior ischemia associated with significant LAD lesion, $77.4 \%$ in determining inferior ischemia associated with significant RCA lesion, and $44.4 \%$ in determining lateral ischemia associated with significant $\mathrm{CX}$ lesion.

Conclusions: Our findings have shown that MPI with visual assessment has $86 \%$ sensitivity for detecting significant coronary artery stenosis. However, the sensitivity of MPI in determining ischemia in the left ventricle region which is supplied by the lesioned coronary artery was found to be 44.4 to $77.4 \%$.

Keywords: myocardial perfusion imaging, coronary angiography, obstructive coronary artery lesion

$\mathrm{C}$ oronary artery disease (CAD) has remained the leading causes of morbidity and mortality all over the world during the last five decades [1]. The initial diagnostic evaluation of patients presenting with chest pain and suspected obstructive CAD generally consists of an exercise electrocardiogram that has a sensitivity of $68 \%$ and a specificity of $77 \%$ in diagnosing obstructive CAD [2]. Nevertheless, the information obtained from the myocardial perfusion imaging (MPI) is more precise with respect to localization, extension, and severity of ischemia; thus it becomes a dominant non-invasive modality, usually a 
gatekeeper test before coronary angiography, for the assessment of patients with suspected CAD in daily practice [3-4].

The performance of the MPI in diagnosing obstructive CAD depends on not only the prevalence of disease in the population studied but also individual characteristics of patients (i.e. likelihood of obstructive CAD) [4]. Thus clinical registries which weigh the performance of MPI among national cohort of patients provide important insights into the real-life health care.

The purpose of the present study was to assess the agreement between MPI and coronary angiography in the identification of the obstructive coronary lesion in patients with suspected CAD. Additionally, we intended to analyze the patient characteristics in whose MPI test was insufficient to detect the obstructive coronary lesions.

\section{METHODS}

\section{Study Population}

In this retrospective study, we initially searched the hospital database to find patients who had undergone both MPI and coronary angiography at our hospital between January 2018 and January 2019. The study protocol was approved by the local ethics committee. Patients who had previously undergone heart surgery, including coronary artery bypass grafting or prosthetic valve replacement and with a prior diagnosis of; heart failure, end-stage renal failure were excluded. Additionally, patients who experienced acute myocardial infarction or undergone revascularization within this period (January 2018 and January 2019), and who had insufficient data on patient cards were excluded. Finally 231 patients who underwent MPI because of clinical suspicion of CAD and were subsequently subjected to a coronary angiogram (owing to ischemia on MPI or ongoing symptoms) were found to be eligible. At this time, MPI records of recruited patients were analyzed to categorize those according to localization of ischemia. Afterwards angiographic findings were analyzed and categorized with respect to lesion localization and being significant $(>70 \%)$ or not. Then data were analyzed to weigh the performance of MPI in determining obstructive coronary lesion. The sensitivity of the MPI was defined as the percentage of any level of ischemia in patients with evidence of obstructive $(>70 \%)$ lesions in one or more coronary arteries. Specificity of the MPI was determined as the percentage of normal images (without ischemia) in patients with normal angiography or with less than $70 \%$ stenosis in any coronary arteries.

Data with respect to demographic and clinical characteristics and laboratory parameters were collected from the medical records. The definition of hypertension was specified as a systolic pressure $\geq$ $140 \mathrm{mmHg}$ and/or a diastolic pressure $\geq 90 \mathrm{mmHg}$ or if the participant was taking an antihypertensive medication [5]. Diabetes mellitus was determined as a fasting glucose level $>126 \mathrm{mg} / \mathrm{dl}$ and/or if the patient was taking an anti-diabetic medication [6]. Participants who were recorded as smoker at the patient card were classified as smokers. Hyperlipidemia was defined as taking a lipid-lowering therapy. A history of CAD was determined as the existence of prior acute coronary syndrome, percutaneous coronary revascularization, and/or at least one attested coronary stenosis $\geq 50 \%$ luminal diameter on angiography [7].

\section{Coronary Angiography Data}

Angiographic data with respect to index coronary angiogram was conducted from the cardiac catheterization laboratory records. Angiographic images were visually assessed by two cardiologists who were blinded to the MPI data. Coronary slow flow phenomenon was defined with respect to corrected TIMI frame count, an objective and quantitative index of coronary flow [8].

\section{Myocardial Perfusion Imaging Data \\ Stress Protocols}

Exercise (treadmill) was the favored stress modality in patients who could exercise and reach sufficient exercise goals. Modified Bruce protocol was used in all of those. Pharmacologic stress with adenosine, dobutamine and dipyridamole was used in patients who could not complete or tolerate sufficient exercise, those with limited heart rate response, or left bundlebranch block. Patients were charged to discontinue taking nitrates for 6 hours, calcium channel blockers for 24 hours, and B-blockers for 48 hours prior to the stress protocol. Tc-99m sestamibi was given through a venous access when the patient's heart rate achieved 
$85 \%$ of calculated maximum heart rate (both in exercise treadmill or pharmacologic stress). Exercise or pharmacological induction was continued for two minutes after the Tc-99m sestamibi injection.

\section{Gated Single-photon Emission Computed Tomogra- phy (SPECT) Protocol}

All patients underwent a Gated SPECT protocol according to current guideline [9]. MPI images were interpreted based on a 17-segment model [10]. All stored images were analyzed by a single, blinded, experienced observer. Images were categorized as either normal or ischemic. A score model was used to define reversible defects. According to this modality, the total score at stress, Summed Stress Score (SSS), reflected the extent and severity of the abnormality including ischemia and infarction. The difference between the SSS and Summed Rest Score (SRS) was defined as Summed Difference Score (SDS) that reflected a reversible defect. Semi-quantitative parameters were classed as follows; A SSS $\leq 3$ was defined as a normal result, while a score of $4-8$ as a mild defect, 9-12 as a moderate defect and $>12$ as a severe defect. A SDS of 1-3 reported mild ischemia, 4-7 moderate ischemia and $>7$ severe ischemia [11].

\section{Statistical Analysis}

The SPSS 22.0 (IBM Corporation, Armonk, New York, USA) was used for statistical analysis. Categorical variables were demonstrated as number and percentage. All quantitative data were expressed as mean \pm SD unless otherwise stated. We compared continuous variables using student t-test or MannWhitney U test between groups. Categorical variables were summarized as percentages and compared with the Chi-square test. Diagnostic concordance with coronary angiogram and MPI data was analyzed was by using cross tabulations. A $p$ value $<0.05$ was considered as statistically significant.

\section{RESULTS}

Two hundred thirty-one patients were included in the present study. Baseline characteristics of the recruited patients are shown in Table 1 . The mean age was $63.9 \pm 8.9$ years (55\% males). $123(53.2 \%)$ patients had non-significant coronary lesions, 54

\section{Table 1. Baseline characteristics of the study patients}

\begin{tabular}{|c|c|}
\hline Parameters & $\mathbf{n}=\mathbf{2 3 1}$ \\
\hline Age (years) & $63.9 \pm 8.9$ \\
\hline Males & $126(54.5 \%)$ \\
\hline History of CAD & $127(54.9 \%)$ \\
\hline Diabetes mellitusy & $97(41.9 \%)$ \\
\hline Hypertension & $208(90 \%)$ \\
\hline Atrial Fibrillation & $16(6.9 \%)$ \\
\hline Hyperlipidemia & $125(54.1 \%)$ \\
\hline \multicolumn{2}{|l|}{ Laboratory parameters } \\
\hline Hemoglobin $(g / L)$ & $13.8 \pm 1.6$ \\
\hline Low-density lipoprotein (mg/dl) & $124.3 \pm 44.7$ \\
\hline Creatinine (mg/dl) & $0.95 \pm 0.57$ \\
\hline Ejection Fraction on the Echocardiography (\%) & $56.6 \pm 9.2$ \\
\hline \multicolumn{2}{|l|}{ Indication of the Myocardial Perfusion Imaging } \\
\hline Angina & $164(70.9 \%)$ \\
\hline $\begin{array}{l}\text { Searching ischemia (e.g. left branch bundle block, any symptom thought to } \\
\text { be an equivalent of angina) }\end{array}$ & $67(29 \%)$ \\
\hline
\end{tabular}


Table 2. Diagnostic concordance between coronary angiography and myocardial perfusion imaging with respect to left anterior descending artery lesions

\begin{tabular}{lccc}
\hline & $\begin{array}{c}\text { Patients without } \\
\text { significant LAD stenosis } \\
(<\mathbf{7 0 \% )} \text { at angiography } \\
(\mathbf{n}=\mathbf{1 7 1 )}\end{array}$ & $\begin{array}{c}\text { Patients with LAD } \\
\text { significant stenosis }(> \\
\mathbf{7 0 \% )} \text { at angiography } \\
(\mathbf{n = 6 0 )}\end{array}$ & p value \\
\hline No ischemia at anterior wall & $78(45.6 \%)$ & $24(40 \%)$ & 0.397 \\
Mild ischemia at anterior wall & $57(33.3 \%)$ & $22(36.6 \%)$ & \\
Moderate ischemia at anterior wall & $32(18.7 \%)$ & $10(16.6 \%)$ & \\
Severe ischemia at anterior wall & $4(2.3 \%)$ & $4(6.6 \%)$ & \\
\hline
\end{tabular}

Data are expressed as percentage (\%) for categorical variables. LAD = left anterior descending artery

(23.3\%) patients had a significant stenosis in only one coronary artery, 38 (16.4\%) patients had significant coronary lesions in two different coronary arteries, while $16(6.9 \%)$ patients had three-vessel coronary artery disease. There were $14(6 \%)$ patients with coronary slow flow who had not a significant lesion in any coronary arteries. A diaphragmatic attenuation was reported in $20(8.6 \%)$ patients. Overall, MPI could identify $86 \%$ of patients who had a significant coronary lesion among our study population.

In the present study, coronary angiography showed a significant $(>70 \%)$ stenosis of LAD in 60 patients. However, MPI did not report any level of ischemia at the anterior wall in $24(40 \%)$ of those, while mild ischemia at the anterior wall was reported in $22(36.6 \%)$ of those. Moderate and severe ischemia at the anterior wall were found to be in $10(16.6 \%)$ and $4(6.6 \%)$ of those, respectively. On the other hand, coronary angiography revealed a no or non-significant stenosis of LAD in 171 patients. MPI did not report any level of ischemia at the anterior wall in 78 $(45.6 \%)$ of those. But, mild, moderate and severe ischemia at the anterior wall were reported in 57 $(33.3 \%), 32(18.7 \%)$, and $4(2.3 \%)$ of those, respectively (Table 2). Thus, sensitivity and specifity of MPI in identifying anterior ischemia associated with critical LAD lesion were found to be $60 \%$ and $45.6 \%$, respectively.

Coronary angiography showed a significant (> $70 \%$ ) stenosis of RCA in 62 patients. However, MPI did not report any level of ischemia at the inferior wall in $14(22.5 \%)$ of those, while mild ischemia at the inferior wall was reported in $7(11.2 \%)$ of those. Moderate and severe ischemia at the inferior wall were found to be in $29(46.7 \%)$ and $12(19.3 \%)$ of those, respectively. On the other hand, coronary angiography revealed a no or non-significant stenosis of RCA in 169 patients. MPI did not report any level of ischemia

Table 3. Diagnostic concordance between coronary angiography and myocardial perfusion imaging with respect to right coronary artery lesions

\begin{tabular}{lccc}
\hline & $\begin{array}{c}\text { Patients without } \\
\text { significant RCA stenosis } \\
(<\mathbf{7 0 \% )} \text { at angiography } \\
(\mathbf{n = 1 6 9 )}\end{array}$ & $\begin{array}{c}\text { Patients with significant } \\
\text { RCA stenosis (> 70\%) } \\
\text { at angiography } \\
(\mathbf{n}=\mathbf{6 2})\end{array}$ & p value \\
\hline No ischemia at inferior wall & $72(42.6 \%)$ & $14(22.5 \%)$ & $<0.001$ \\
$\begin{array}{l}\text { Mild ischemia at inferior wall } \\
\begin{array}{l}\text { Moderate ischemia at inferior } \\
\text { wall }\end{array}\end{array}$ & $25(14.7 \%)$ & $7(11.2 \%)$ & \\
Severe ischemia at inferior wall & $68(40.2 \%)$ & $29(46.7 \%)$ & \\
\hline
\end{tabular}

Data are expressed as percentage $(\%)$ for categorical variables. $\mathrm{RCA}=$ right coronary artery 


\begin{tabular}{lccc}
$\begin{array}{l}\text { Table 4. Diagnostic concordance } \\
\text { perfusion imaging with respect to circumflex artery lesions }\end{array}$ & $\begin{array}{c}\text { Petween coronary } \\
\text { Patients without } \\
\text { significant CX stenosis } \\
(<\mathbf{7 0 \% )} \text { at angiography } \\
(\mathbf{n = 1 7 7 )}\end{array}$ & $\begin{array}{c}\text { Patients with significant } \\
\text { CX stenosis }(>\mathbf{7 0} \%) \text { at } \\
\text { angiography } \\
(\mathbf{n}=\mathbf{5 4})\end{array}$ & p value \\
\hline No ischemia at lateral wall & $127(71.7 \%)$ & $30(55.5 \%)$ & 0.001 \\
\hline Mild ischemia at lateral wall & $17(9.6 \%)$ & $3(5.5 \%)$ & \\
Moderate ischemia at lateral wall & $31(17.5 \%)$ & $15(27.7 \%)$ & \\
\hline Severe ischemia at lateral wall & $2(1.1 \%)$ & $6(11.1 \%)$ & \\
\hline
\end{tabular}

Data are expressed as percentage $(\%)$ for categorical variables. $\mathrm{CX}=$ circumflex artery

at the inferior wall in $72(42.6 \%)$ of those. But, mild, moderate and severe ischemia at the inferior wall were reported in $25(14.7 \%), 68(40.2 \%)$, and $4(2.3 \%)$ of those, respectively (Table 3 ). Thus, sensitivity and specifity of MPI in identifying inferior ischemia associated with critical RCA lesion were found to be 77.4 $\%$ and $42.6 \%$, respectively.

Additionally, coronary angiography showed a significant $(>70 \%)$ stenosis of $\mathrm{CX}$ in 54 patients. However, MPI did not report any level of ischemia at the lateral wall in $30(55.5 \%)$ of those, while mild ischemia at the lateral wall was reported in $3(5.5 \%)$ of those. Moderate and severe ischemia at the lateral wall were found to be in $15(27.7 \%)$ and $6(11.1 \%)$ of those, respectively. On the other hand, coronary angiography revealed a no or non-significant stenosis of CX in 177 patients. MPI did not report any level of ischemia at the lateral wall in $127(71.7 \%)$ of those. But, mild, moderate and severe ischemia at the lateral wall were reported in $17(9.6 \%), 31(17.5 \%)$, and 2 (1.1\%) of those, respectively (Table 4$)$. Thus, sensitivity and specifity of MPI in identifying lateral ischemia associated with critical CX lesion were found to be $44.4 \%$ and $71.7 \%$, respectively.

While evaluating overall (regardless of the relationship between the vessel and its feeding area) sensitivity, MPI could identify; $85 \%$ of patients who had a significant LAD lesion, $88 \%$ of patients who had a significant RCA lesion, and $83 \%$ of patients who had a significant $\mathrm{CX}$ lesion.

While assessing patients with moderate-severe ischemia at the anterior wall $(n=50)$, there were not any significant difference on baseline characteristics with respect to lesion severity in LAD. However among patients with moderate-severe ischemia at the inferior wall $(n=113)$, subjects who had a significant lesion on RCA were more likely to have a prior history of diabetes mellitus, coronary artery disease and be male ( 48.7 vs. $29.1 ; p=0.042,78 \%$ vs. $56.9 \%$; $p=$ $0.024,9.7 \%$ vs. $38.8 \% ; p<0.001$, respectively).

Coronary angiography demonstrated a significant $(>50 \%)$ stenosis of LMCA in 4 patients. One of them displayed moderate-severe ischemia at the anterior wall on the MPI; one of them displayed moderatesevere ischemia at the lateral wall while the other two patients revealed moderate-severe ischemia at the inferior wall.

\section{DISCUSSION}

The present study was an evaluation of patient characteristics and the agreement between MPI and coronary angiography in the identification of the obstructive coronary lesion $(>70 \%)$ in patients with suspected CAD admitted to a tertiary referral hospital. The main results of the present study were as follows: 1) MPI showed a sensitivity of 0.86 in determining patients who had a significant coronary lesion; 2) The overall sensitivity was found to be 0.85 in determining significant LAD lesion while anterior ischemia associated with significant LAD lesion MPI showed a sensitivity of $0.6 ; 3$ ) The overall sensitivity was found to be 0.88 in determining significant RCA lesion while inferior ischemia associated with significant RCA lesion MPI showed a sensitivity of 0.77 ; 4) The over- 
all sensitivity was found to be 0.83 in determining significant CX lesion while lateral ischemia associated with significant $\mathrm{CX}$ lesion MPI showed a sensitivity of 0.44).

Modern cardiology has a broad range of clinical exploration methods available to assist the diagnosis and to stratify the risk of patients with suspected coronary artery disease. Basic (first-line) testing in those includes standard laboratory biochemical testing, a resting ECG, possible ambulatory ECG monitoring, and structural tests including resting echocardiography, and, in selected patients, cardiac magnetic resonance (CMR) [4]. In patients, with an intermediate probability of $\mathrm{CAD}$ and need to be tested noninvasively, an exercise ECG is the most widely used non-invasive method [12]. Although exercise ECG has advantages, easy to access and cheaper test comparing with the other non-invasive test options (stress CMR or stress echocardiography or MPI), because of the moderate diagnostic accuracy of it, clinicians usually could not be sure while excluding $\mathrm{CAD}$ with the result of the exercise ECG alone. Moreover, The National Institute for Health and Care Excellence (NICE) has recommended that exercise ECG testing should not be used alone for the diagnosis (or exclusion) of stable angina in patients without known CAD [13]. This recommendation has been followed in part by the latest European Society of Cardiology guideline on the management of chronic coronary syndromes, which suggests the use of functional imaging (stress CMR or stress echocardiography or MPI) over exercise ECG testing in patients with suspected CAD, in whom the results of exercise ECG are inadequate to diagnose or exclude CAD [4]. Although Stress CMR has some technical advantages, it is not easily accessible, it is contraindicated in patients who either have a pacemaker or severe renal failure, it is very sensitive to the rhythm of the heart thus it may not be interpretable in patients with atrial fibrillation [14]. On the other hand results of the stress echocardiography is usually challenging in patients with left bundle branch block or septal dyssynchrony [15]. As a result of those, cardiologists usually order MPI as a first-step test, even it is not recommended at this stage, in most of patients with chest pain admitted to cardiology department [12]. Thus the performance of the MPI, not only in recognizing coro- nary artery disease but also in detecting severe lesion, is important in daily practice. Previous studies evaluating the concordance of findings between coronary angiography and MPI reported different accuracy rates which were between $41.7 \%$ and $93.3 \%$ [16-17]. Hasbek et al. revealed a slightly low, $41.7 \%$, diagnostic concordance between coronary angiography and MPI, in which treadmill test was the favored stress modality. However, they reported a $75.8 \%$ concordance rate in patients whom stress modality was a pharmacologic stress with adenosine [16]. Thus diagnostic accuracy of MPI depends on studied population and design of the works. Many of the previous studies defined culprit coronary lesion as $50 \%$ or more stenosis responsible for the symptoms while evaluating the concordance between coronary angiography and MPI. In other words, these studies were design to evaluate the power of MPI in detecting a coronary stenosis of $50 \%$ or above [16-20]. However current data has clearly revealed that only an estimated diameter stenosis $>70 \%$ in a coronary artery is classified as severe lesion and predicted to cause symptoms. Thus in the present study we aimed to evaluate the performance of MPI in determining a coronary stenosis $>70 \%$.

In the present study, MPI could identify $86 \%$ of patients who had a significant coronary lesion that was very similar to previous studies $[18,21]$. However while determining the lesion associated ischemic zone, sensitivity rates were fallen (e.g. 77\% for RCA associated inferior ischemia, $60 \%$ for LAD associated anterior ischemia, and $44 \%$ for CX lesion associated lateral ischemia). This means that seven of every 100 patients with critical stenosis in RCA had an ischemia on another wall than inferior, 26 of every 100 patients with critical stenosis in LAD had an ischemia on another wall than anterior, and 42 of every 100 patients with critical stenosis in CX had an ischemia on another wall than lateral. The possible explanations for these findings are: I) existing collateral circulation; II) variation in coronary anatomy; III) heterogeneity of coronary hyperemia with pharmacologic stress [22]; IV) technical issues (As example; in normal SPECT images, the lateral wall often may appear brighter than the contra-lateral septum because of the camera is physically closer to the lateral myocardial wall (in proximity to the lateral chest wall) than to the septum. So that, the acquisition is associated with 
more efficient count capture [22] - this may explain why MPI much more deviated in revealing lateral wall ischemia associated with CX lesion).

One of the remarkable findings of the present study was that patients who had a ischemia at the inferior wall, even if moderate, the possibility of critical stenosis in RCA was found to be considerably decreased if they were female or had not a prior history of diabetes mellitus or coronary artery disease. This finding is important because, physicians are everyday faced with such cases. The possible explanations for this finding are; I) breast attenuation; II) it is plausible that females who have a diabetes mellitus or coronary artery disease are more likely to be overweight and have large breasts that could affect the performance of MPI, in particular in evaluating inferior wall perfusion. Further studies need to be carried out in order to validate this finding.

\section{Limitations}

Our study has several limitations. First, we analyzed MPI images with visual analysis. Indeed published data has not clearly demonstrated improved sensi $\neg$ tivity or specificity of automated quantitative analysis systems programs over visual analysis. Moreover the visual analysis data are derived from experienced readers in laboratories have been found to be an excellent quality control [22]. However it would be better if every single patient were analyzed with an automated quantitative analysis program. Second, we did not analyze the results with respect to sub-group according to modality of stress (pharmacologic or treadmill) on MPI. Third, the present work is a single-center analysis; therefore, the generalisability of these findings is limited. Fourth, we did not evaluate the outcome of patients. Finally, we cannot exclude the possibility of unmeasured confounding factors.

\section{CONCLUSION}

In conclusion, the present study is an evaluation of patient characteristics and the agreement between MPI and coronary angiography in the identification of the obstructive coronary lesion $(>70 \%)$ in patients with suspected CAD. Our findings have shown that MPI with visual assessment has $86 \%$ sensitivity for detecting significant coronary artery stenosis. However, the sensitivity of MPI in determining ischemia in the left ventricle region which is supplied by the lesioned coronary artery was found to be 44.4 to $77.4 \%$.

\section{Conflict of interest}

The author disclosed no conflict of interest during the preparation or publication of this manuscript.

\section{Financing}

The authors disclosed that they did not receive any grant during conduction or writing of this study.

\section{REFERENCES}

1. World Health Organization, Top 10 causes of death. http://www9.who.int/gho/mortality_burden_disease/causes_deat h/top_10/en/

2. Detrano R, Gianrossi R, Froelicher V. The diagnostic accuracy of the exercise electrocardiogram: a metaanalysis of 22 years of research. Prog Cardiovasc Dis 1989;32:173-206.

3. Candell-Riera J, Santana-Boado C, Castell-Conesa J, AguadéBruix S, Olona-Cabases M, Domingo E, et al. Culprit lesion and jeopardized myocardium: correlation between coronary angiography and single-photon emission computed tomography. Clin Cardiol 1997;20:345-50.

4. Knuuti J, Wijns W, Saraste A, Capodanno D, Barbato E, Funck-Brentano C, et al. 2019 ESC Guidelines for the diagnosis and management of chronic coronary syndromes. Eur Heart J 2020;41:407-77.

5. Williams B, Mancia G, Spiering W, Rosei EA, Azizi M, Burnier M, et al; ESC Scientific Document Group. 2018 ESC/ESH Guidelines for the management of arterial hypertension: The Task Force for the management of arterial hypertension of the European Society of Cardiology (ESC) and the European Society of Hypertension (ESH). Eur Heart J 2018;39:3021-104. 6. Cosentino F, Grant PJ, Aboyans V, Bailey CJ, Ceriello A, Delgado V, et al. 2019 ESC Guidelines on diabetes, pre-diabetes, and cardiovascular diseases developed in collaboration with the EASD: The Task Force for diabetes, pre-diabetes, and cardiovascular diseases of the European Society of Cardiology (ESC) and the European Association for the Study of Diabetes (EASD). Eur Heart J 2020;41:255-323.

7. Schuijf JD, Wijns W, Jukema JW, Atsma DE, de Roos A, Lamb HJ, et al. Relationship between noninvasive coronary angiography with multi-slice computed tomography and myocardial perfusion imaging. J Am Coll Cardiol 2006;48:2508-14.

8. Gibson CM, Cannon CP, Daley WL, Dodge JT Jr, Alexander B Jr, Marble SJ, et al. TIMI frame count: a quantitative method of assessing coronary artery flow. Circulation 1996;93:879-88. 9. Verberne HJ, Acampa W, Anagnostopoulos C, Ballinger J, Bengel F, De Bondt P, et al; European Association of Nuclear 
Medicine (EANM). EANM procedural guidelines for radionuclide myocardial perfusion imaging with SPECT and SPECT/CT: 2015 revision. Eur J Nucl Med Mol Imaging 2015;42:1929-40.

10. Cerqueira MD, Weissman NJ, Dilsizian V, Jacobs AK, Kaul S, Laskey WK, et al; American Heart Association Writing Group on Myocardial Segmentation and Registration for Cardiac Imagin. Standardized myocardial segmentation and nomenclature for tomographic imaging of the heart. A statement for healthcare professionals from the Cardiac Imaging Committee of the Council on Clinical Cardiology of the American Heart Association. Int J Cardiovasc Imaging 2002;18:539-42.

11. IAEA Human Health Series No. 23 (Rev 1) Nuclear Cardiology: Guidance on the Implementationof SPECT Myocardial Perfusion Imaging International Atomic Agency, Vienna; 2016. 12. Vrints CJ. Refined interpretation of exercise ECG testing: Opportunities for a comeback in the era of expanding advanced cardiac imaging technologies? Eur J Prev Cardiol 2016;23:162831 .

13. National Clinical Guideline Centre for Acute and Chronic Conditions (UK). Chest Pain of Recent Onset: Assessment and Diagnosis of Recent Onset Chest Pain or Discomfort of Suspected Cardiac Origin [Internet]. London: Royal College of Physicians (UK); March 2010.

14. Rieber J, Huber A, Erhard I, Mueller S, Schweyer M, Koenig A, et al. Cardiac magnetic resonance perfusion imaging for the functional assessment of coronary artery disease: a comparison with coronary angiography and fractional flow reserve. Eur Heart J 2006;27:1465-71.

15. Pellika PA, Nagueh SF, Elhendy AA, Kuehl CA, Sawada SG, American Society of Echocardiography. American Society of Echocardiography recommendations for performance, interpretation, and application of stress echocardiography. J Am Soc
Echocardiogr 2007;20:1021-41.

16. Hasbek Z, Ertürk SA, Çakmakçılar A, Gül İ, Yılmaz A. Evaluation of myocardial perfusion imaging SPECT parameters and pharmacologic stress test with adenosine versus coronary angiography findings: are they diagnostically concordant? Mol Imaging Radionucl Ther 2019;28:53-61.

17. Yao Z, Liu XJ, Shi RF, Dai R, Zhang S, Liu YZ, et al. A comparison of 99Tcm-MIBI myocardial SPET and electron beam computed tomography in the assessment of coronary artery disease in two different age groups. Nucl Med Commun 2000;21:43-8.

18. Gonzalez P, Massardo T, Jofre MJ, Yovanovich J, Prat H, Munoz A, et al. 201Tl myocardial SPECT detects significant coronary artery disease between $50 \%$ and $75 \%$ angiogram stenosis. Rev Esp Med Nucl 2005;24:305-11.

19. Johansen A, Hoilund-Carlsen PF, Christensen HW, Vach W, Jorgensen HB, Veje A, et al. Diagnostic accuracy of myocardial perfusion imaging in a study population without post-test referral bias. J Nucl Cardiol 2005;12:530-7.

20. Schepis T, Gaemperli O, Koepfli P, Namdar M, Valenta I, Scheffel H, et al. Added value of coronary artery calcium score as an adjunct to gated SPECT for the evaluation of coronary artery disease in an intermediate-risk population. J Nucl Med 2007;48:1424-30.

21. Jeetley P, Hickman M, Kamp O, Lang RM, Thomas JD, Vannan MA, et al. Myocardial contrast echocardiography for the detection of coronary artery stenosis: a prospective multicenter study in comparison with single-photon emission computed tomography. J Am Coll Cardiol 2006;47:141-5.

22. Udelson JE, Dilsizian V, Bonow RO. Nuclear Cardiology. In: Eugene Braunwald, eds. Braunwald's Heart Disease a Textbook of Cardiovascular Medicine. PA, Elsevier Inc.; 2015:271-316. 